\title{
Abnormal Sequencing of Motor Actions in Patients With Schizophrenia: Evidence From Grip Force Adjustments During Object Manipulation
}

Yvonne Delevoye-Turrell, Ph.D.

Anne Giersch, Ph.D.

Jean-Marie Danion, M.D.
Objective: During object manipulation, grip force must be adjusted in anticipation of destabilizing load forces to prevent the object from slipping. This study used three gripping tasks to assess whether schizophrenia affects the predictive mechanisms required for the scaling, timing, and/or sequencing of motor actions.

Method: Sixteen patients with schizophrenia and 16 comparison subjects matched for age, sex, and educational level 1) lifted objects of various mass and texture (lift task) and 2) used a manipulandum to hit (hit task) or resist (resist task) impacts produced by a collision with a pendulum. For an optimized performance, all tasks demanded a predictive increase in grip force and the timing of external events. The fluid sequencing of finger and hand submovements was required for the lift and the hit tasks but not for the resist task.

Results: Patients were impaired in the smooth execution of both the lift and the hit tasks but not in the performance of the resist task. In all three tasks, the scaling of grip force was similar for patients and comparison subjects.

Conclusions: Schizophrenia is associated with a specific deficit in the sequencing of motor actions rather than with an overall problem in the predictive control of movement.

(Am J Psychiatry 2003; 160:134-141)

A Imost a century ago, Bleuler (1) and Kraepelin (2) described multiple motor features in the majority of their patients with schizophrenia. These features included irregular timing and spacing of steps during gait, as well as reduced efficiency and coordination of fine movements during handiwork and crafts. Clinical reports later described deficient motor synchrony as well as generalized incoordination and clumsiness in voluntary motor behavior (see Boks et al. [3], for a review). These motor deficits appeared to be relatively independent of cognitive impairments (4). However, the exact nature and cause of the motor perturbations remain unclear.

The combined role of anticipatory and reactive mechanisms in the generation of purposeful motor output has now been well established. Because of its inherent time delays, the motor system can not solely depend on reactive mechanisms (5). Consequently, anticipatory control is thought to be crucial for skilled motor performance (6). It has been suggested that schizophrenia is a pathology specifically affecting certain predictive mechanisms involved in the anticipatory control of motor actions $(7,8)$. This study was conducted to establish which aspects of these predictive adjustments may be impaired during object manipulation in patients with schizophrenia.

A lift task was used to test the predictive mechanisms required for the scaling, timing, and sequencing of volun- tary motor activity. To lift an object, the grip force applied to the surfaces of the object must result in sufficient friction to overcome the destabilizing load force due to gravity. In subjects with intact anticipatory mechanisms, grip force is tightly scaled both to the mass and to the texture of manipulated objects. Hand transport, grasp closure, and the upward lift movement are also well coordinated, with grip force peak occurring at the moment of object liftoff (9).

The duration of a lift is of the order of several hundred milliseconds, and hence, it is long enough to allow both anticipatory and reactive mechanisms to act in a complementary manner to ensure grasp stability. In the event of a collision, there is no time for reactive feedback correction (10). Hence, two collision tasks - previously developed for healthy comparison subjects (11)-were used to examine the working state of the predictive mechanisms in patients with schizophrenia. Subjects used a manipulandum to either produce or resist an imposed collision with a pendulum. In the task in which a collision was produced (hit task), good performance required scaling, timing, and sequencing of upper limb movement; the resist task demanded only scaling and timing of motor action. Combining the results of the lift, hit, and resist tasks provided a means of isolating specific deficits in the sequencing of motor actions in patients with schizophrenia. 


\section{Method}

\section{Participants}

Sixteen patients with schizophrenia (seven female and nine male subjects; mean age $=37.6$ years, $\mathrm{SD}=15.5$, range $=18-50$; mean educational level $=12.1$ years, $\mathrm{SD}=7.0$, range $=6-20$ ) and 16 healthy comparison subjects (seven female and nine male subjects; mean age $=39.0$ years, $S D=16.5$, range $=19-50$; mean educational level $=$ 12.8 years, $\mathrm{SD}=6.2$, range $=8-20$ ) provided written informed consent and were paid for their participation.

Psychiatric diagnoses of the patients with schizophrenia were established in accordance with their referring therapist and fulfilled the DSM-IV criteria for schizophrenia. The mean age at onset of schizophrenia symptoms was 24.7 years $(\mathrm{SD}=7.9$, range $=10$ 38 ), the mean disease duration was 14.5 years ( $\mathrm{SD}=7.0$, range $=7-$ 27 ), and the mean number of hospitalizations was 6.5 ( $\mathrm{SD}=6.5$, range $=0-20$ ). Patients with schizophrenia were clinically stable and underwent clinical assessment. The mean level of symptom severity, as measured by the Brief Psychiatric Rating Scale total score, was 40.5 ( $\mathrm{SD}=18.3$, range $=14-74)$, with higher scores reflecting greater symptom severity. The mean scores on the Scale for the Assessment of Positive and Negative Symptoms were 32.4 ( $\mathrm{SD}=25.9$, range $=2-102)$ for positive symptoms and $41.8(\mathrm{SD}=$ 20.4, range $=7-66$ ) for negative symptoms, with higher scores reflecting greater symptom severity.

Fourteen of the sixteen patients were receiving long-term neuroleptic treatment, administered in a standard dose (mean dose $=$ $244 \mathrm{mg} /$ day of chlorpromazine or chlorpromazine equivalents, $\mathrm{SD}=171)$. Seven were also receiving antiparkinsonian treatment, either trihexyphenidyl (mean dose $=11 \mathrm{mg} /$ day, $\mathrm{SD}=6$ ) or tropatepine (mean dose $=18 \mathrm{mg} /$ day, $\mathrm{SD}=11$ ). Two patients were not receiving any treatment.

The comparison subjects were recruited through local advertisements. Exclusion criteria for both groups were visual and auditory disorders, history of neurological illness or trauma, alcohol or other substance abuse, and age older than 55 years or younger than 18 years. The patient and the comparison groups did not differ significantly in age, sex, or educational level.

\section{Experimental Protocol}

With their dominant hand, subjects used a precision grip between thumb and four fingers to grasp a manipulandum with wood-surfaced sides $(4.5 \times 10.0 \times 9.0 \mathrm{~cm}, 450 \mathrm{~g})$. A six-axis load cell (Novatech Gamma SI-130-10, Novatech Measurements, Ltd., East Sussex, U.K.) was mounted within this manipulandum to measure forces and torques that were normal and tangential to the loading axes. Data were sampled at $1000 \mathrm{~Hz}$.

To examine possible group differences in frictional condition, subjects started the 1-hour session by performing voluntary object slips (five trials). The mean grip force levels when the object started to slip was calculated for each individual. Subjects then performed the three experimental tasks. The lift task was presented first, as it was the most familiar. In randomized fashion, subjects then performed the two collision tasks.

The lift task. Subjects were seated at a table with their dominant hand positioned $40 \mathrm{~cm}$ from the manipulandum. An auditory signal indicated the start of each trial, from which point the subjects' task was to reach for and lift the manipulandum, maintain it at a steady position $20 \mathrm{~cm}$ from the tabletop for approximately 3 seconds, and then place the manipulandum back on the table. Movement speed was not emphasized in the instructions given to the subjects. The properties of the manipulandum were varied in a series of trials. The grasp surface of the manipulandum was covered with increasingly slippery materials (sandpaper, cotton, silk), and iron bars were attached to the bottom of the manip- ulandum to vary the object mass (from light [ $450 \mathrm{~g}]$ to medium $[710 \mathrm{~g}]$ and heavy $[880 \mathrm{~g}]$ ). Subjects were aware of the changes in the object's properties and performed five trials for each of the nine mass/texture combinations, presented in randomized blocks. This procedure allowed assessment of whether patients were able to integrate various intrinsic properties of an object for an optimized adjustment of grip force levels.

Collision tasks. Subjects stood facing a pendulum (an aluminum bar, $75 \mathrm{~cm}$ in length and $750 \mathrm{~g}$ in weight) that swung in the frontoparallel plane. A circular potentiometer located at the pivoting point measured the angle of pendulum swing (in degrees). Subjects gripped the surfaces of the manipulandum, which were covered with cotton fabric, between the thumb and four fingers of their dominant hand.

Hit task. The subject's task was to use the manipulandum to hit the head of the pendulum to make it reach target heights of $5^{\circ}$, $10^{\circ}$, or $18^{\circ}\left( \pm 0.5^{\circ}\right)$. Subjects performed 12 trials for each pendulum target angle, which were presented in a semirandomized order. This task demanded 1) anticipatory scaling of grip force to the magnitude of the collision, 2) fluid sequencing of the onset of the grip force increase with the start of arm movement, and 3) estimation of the time of impact.

Resist task. Subjects held the manipulandum against the head of the pendulum. When they were ready, the experimenter raised the pendulum to a pre-set angle of $6^{\circ}, 12^{\circ}$, and $20^{\circ}\left( \pm 0.5^{\circ}\right)$. These release angles were chosen so that subjects experienced similar forces of impact in both collision tasks. Then, the experimenter released the pendulum at various time intervals $(0.5-2.0$ seconds) after an auditory signal, which indicated the start of the trial. The subjects' task was to maintain the manipulandum immobile throughout the trial. Subjects performed 12 trials for each pendulum release angle. As the arm was immobile throughout, no movement sequencing was involved in this task, and good performance was reached as long as the subject could accurately predict the force and the time of the impact.

\section{Statistical Analysis}

Grip force and load force curves were differentiated to obtain the rate of change of grip force and the rate of change of load force. The results of each trial were scored automatically.

In the lift task (Figure 1, left), the values and the times of peak grip force, rate of change of grip force, load force, and rate of change of load force were measured. The times of onset of the grip force increase and of the load force increase were defined as the moment when their derivatives reached 10 newtons/second $(\mathrm{N} / \mathrm{sec})$. The time of load force peak indicated the moment of object liftoff. The peak rate of change of load force indicated the speed at which the subjects lifted the object. Finally, the mean and the standard deviation of grip force during the 500-msec static hold phase were measured $250 \mathrm{msec}$ after the peak of grip force. A three-way repeated measures analysis of variance (ANOVA) was carried out with group (patient group, comparison group), object texture (sandpaper, cotton, silk), and object mass (light, medium, heavy) as factors.

In the collision tasks (the hit and resist tasks) (Figure 1, right), the mean and standard deviation of the grip force baseline were measured during the first $500 \mathrm{msec}$ of each trial. The values and the times of peak grip force, rate of change of grip force, load force, and pendulum angle were also measured. The time of onset of the grip force increases was defined as in the lift task. The two collision tasks were analyzed separately. For both, a two-way repeated measures ANOVA was carried out with group (patient group, comparison group) and target $\left(5^{\circ}, 10^{\circ}, 18^{\circ}\right.$ for the hit task; $6^{\circ}, 12^{\circ}, 20^{\circ}$ for the resist task) as factors. 
FIGURE 1. Details of the Measurements of Grip Force and Load Force for the Lift and the Collision Tasks Performed by Healthy Comparison Subjects and Patients With Schizophrenia ${ }^{\mathrm{a}}$
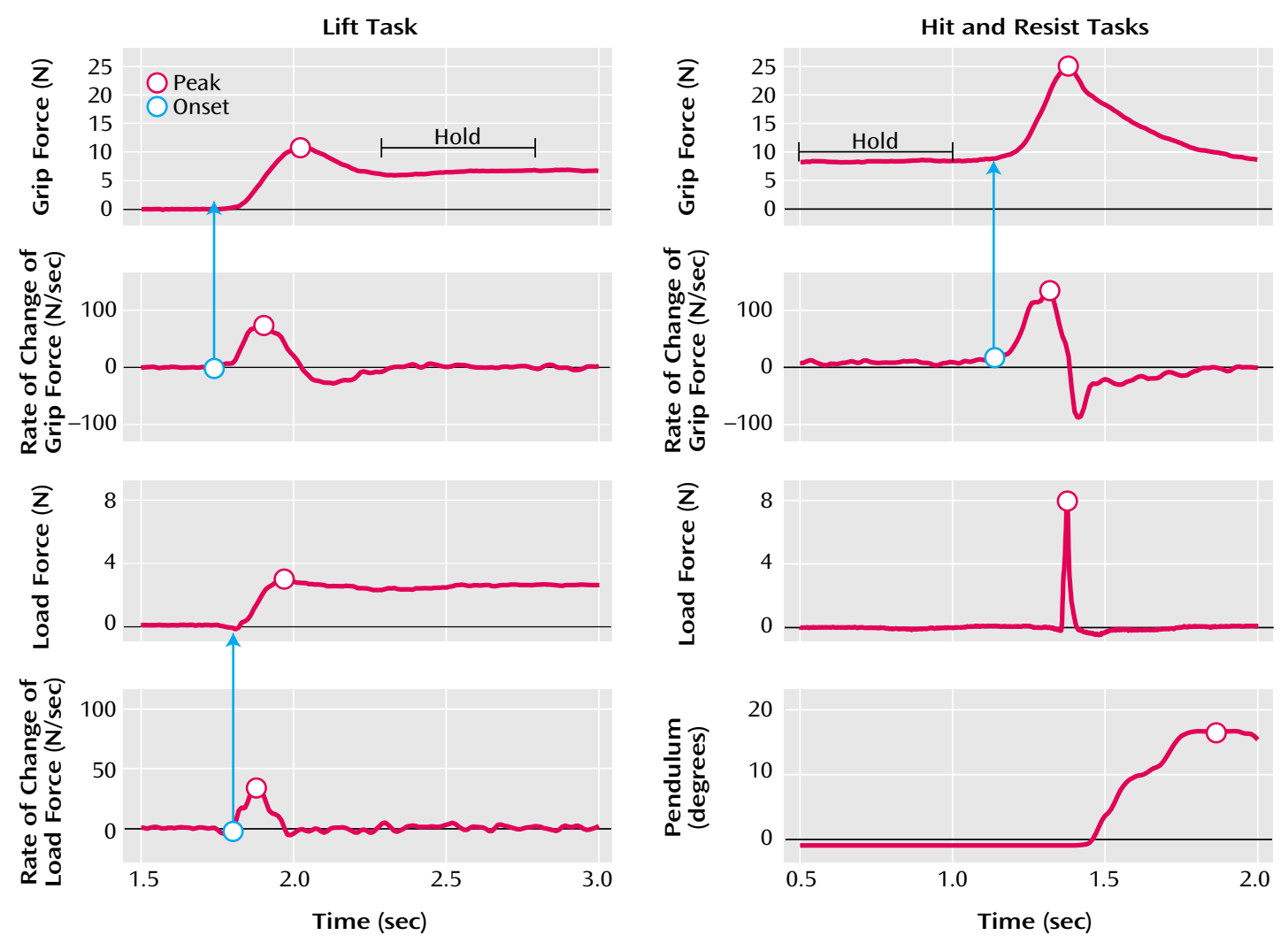

a See text for additional description. Forces measured in newtons $(\mathrm{N})$.

\section{Results}

The patients and comparison subjects performed the tasks without difficulty. Figure 2 shows representative force recordings generated by a comparison subject (top) and a nonmedicated patient with schizophrenia (bottom) while lifting the light cotton-covered object (left), while using the manipulandum to hit the pendulum (center), and while using the manipulandum to resist an imposed collision (right). Results for the medicated and nonmedicated patients were similar in all points. There was no correlation between reported results and the patients' level of medication.

\section{Lift Task}

The measurement of the peak rate of change of load force indicated that the patients (mean $=45 \mathrm{~N} / \mathrm{sec}, \mathrm{SD}=2$ ) and the comparison subjects (mean $=49 \mathrm{~N} /$ second, $\mathrm{SD}=2$ ) lifted the objects at a similar speed $(\mathrm{F}=1.31, \mathrm{df}=1,133$, n.s.) (Table 1). In the comparison subjects, grip force and load force curves rose in parallel and peaked in synchrony, which suggested that the arm movement, the grip closure, and the start of the upward arm movement were well sequenced (Figure 2, top left). In the patients with schizophrenia, the force patterns were different (Figure 2, bottom left). Patients took more time to establish a firm grip

and frequently tended to push down on the object before initiating the lift.

The statistical analysis confirmed the previous observations by indicating that the time from the onset of grip force increase to the onset of load force increase was significantly longer for the patients than for the comparison subjects $(\mathrm{F}=26.08, \mathrm{df}=1,133, \mathrm{p}<0.01)$. The time from load force peak to grip force peak was, however, similar for the patients and the comparison subjects $(\mathrm{F}=0.42, \mathrm{df}=1,133$, n.s.). The intrinsic properties of the object had no selective group effect on the coordination of grip force and load force patterns.

For all subjects, the grip force peak was scaled to the texture $(\mathrm{F}=289.55, \mathrm{df}=2,266, \mathrm{p}<0.01)$ and to the mass $(\mathrm{F}=$ $370.43, \mathrm{df}=2,266, \mathrm{p}<0.01$ ) of the manipulandum: grip force peak was scaled higher for the objects that were more slippery and heavier. The peak rate of change of grip force was also scaled to the texture $(\mathrm{F}=122.80, \mathrm{df}=2,266, \mathrm{p}<0.01)$ and to the mass of the manipulandum $(\mathrm{F}=63.17, \mathrm{df}=2,266$, $\mathrm{p}<0.01$ ), which indicated that all subjects increased grip force at a faster rate when planning to lift heavier or more slippery objects. Finally, grip force levels remained scaled to the texture $(\mathrm{F}=326.10, \mathrm{df}=2,266, \mathrm{p}<0.01)$ and to the mass ( $F=722.54, \mathrm{df}=2,266, \mathrm{p}<0.01)$ of the manipulandum during the static hold phase of the task (Figure 3). 
FIGURE 2. Representative Grip Force and Load Force Recordings for a Healthy Comparison Subject and a Nonmedicated Patient With Schizophrenia Who Completed the Lift, the Hit, and the Resist Tasks ${ }^{\mathrm{a}}$
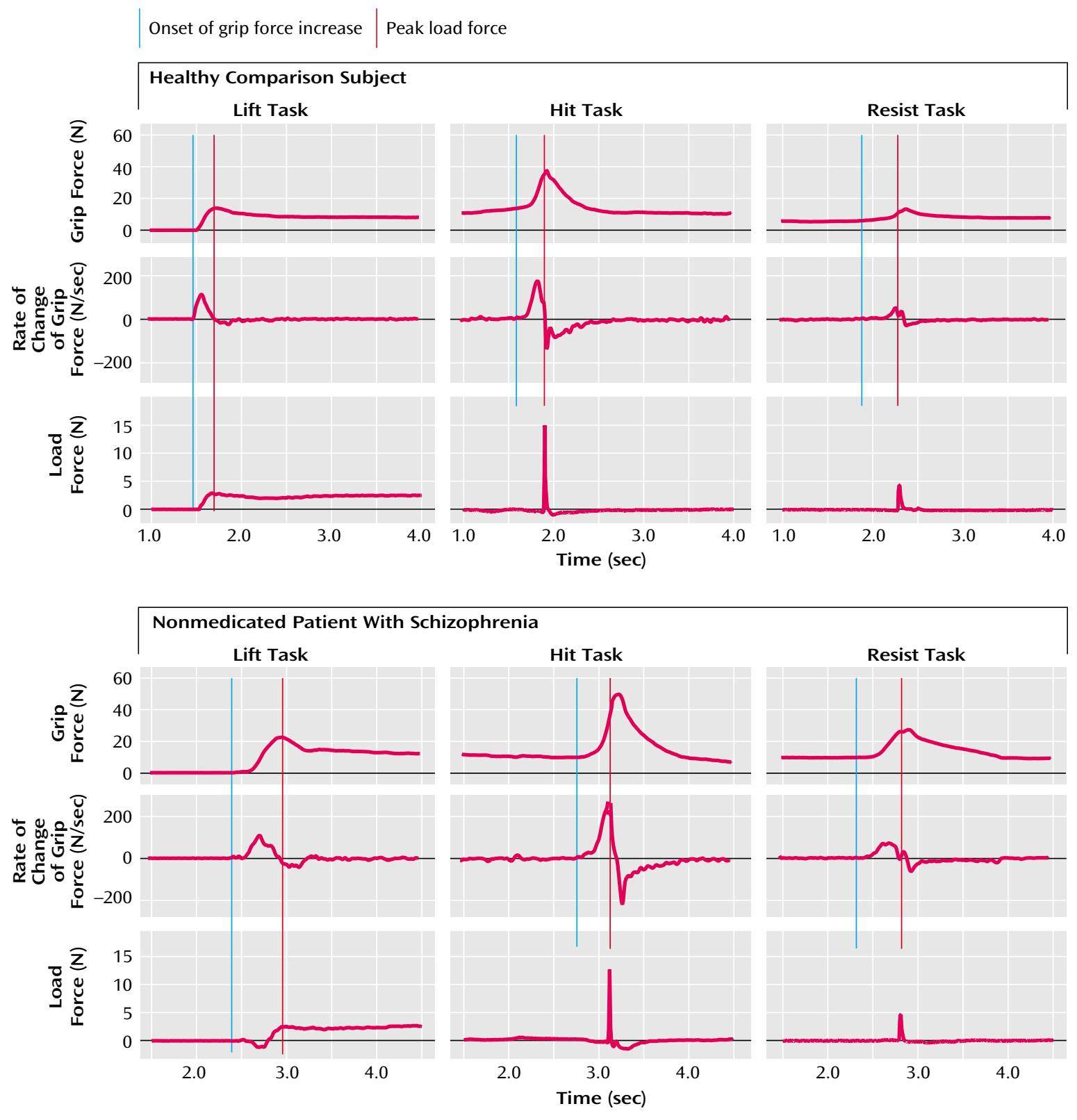

${ }^{\text {a }}$ Forces measured in newtons $(\mathrm{N})$.

For the same object, the grip force peak was significantly larger for patients than for comparison subjects $(\mathrm{F}=8.29$, $\mathrm{df}=1,133, \mathrm{p}<0.01)$. This difference was not due to a difference in the predictive control of grip force, as the peak rates of change of grip force were similar in both groups $(\mathrm{F}=0.27$, $\mathrm{df}=1,133$, n.s.). During static hold, grip force levels were both greater $(\mathrm{F}=7.47, \mathrm{df}=1,133, \mathrm{p}<0.01)$ and more variable $(\mathrm{F}=19.06, \mathrm{df}=1,133, \mathrm{p}<0.01)$ for patients than for comparison subjects. As the minimum grip force required to prevent the object from slipping was similar for the patients (mean $=5.9 \mathrm{~N}, \mathrm{SD}=0.4$ ) and for the comparison subjects (mean=6.2 N, SD=0.4) $(\mathrm{F}=0.38, \mathrm{df}=1,170$, n.s.), the differ- ence in grip force levels during object manipulation was not due to a group difference in the slip ratio.

\section{Hit Task}

The force patterns were similar for the patients and the comparison subjects (Table 2; Figure 2, center). Typically, subjects had a steady grip force baseline while waiting for the auditory tone. As they started to move the hand, grip force increased and reached a maximum value close to the time of impact. As a result of the impact, the pendulum started to swing (Figure 1, right). The maximum pendulum angle was on average within $1.8^{\circ}\left(\mathrm{SD}=0.6^{\circ}\right)$ of target values for the patients and within $2.2^{\circ}\left(\mathrm{SD}=0.6^{\circ}\right)$ for the 
TABLE 1. Measures of Grip Force and Load Force for 16 Healthy Comparison Subjects and 16 Patients With Schizophrenia Who Completed a Lift Task Requiring the Integration of Information About an Object's Texture and Mass ${ }^{\mathrm{a}}$

\begin{tabular}{|c|c|c|c|c|c|c|c|c|c|c|c|c|}
\hline \multirow[b]{3}{*}{ Measure and Group } & \multicolumn{6}{|c|}{ Object Texture } & \multicolumn{6}{|c|}{ Object Mass $^{\mathrm{b}}$} \\
\hline & \multicolumn{2}{|c|}{ Sandpaper } & \multicolumn{2}{|c|}{ Cotton } & \multicolumn{2}{|c|}{ Silk } & \multicolumn{2}{|c|}{ Light } & \multicolumn{2}{|c|}{ Medium } & \multicolumn{2}{|c|}{ Heavy } \\
\hline & Mean & SD & $\overline{\text { Mean }}$ & SD & Mean & SD & Mean & SD & Mean & SD & Mean & SD \\
\hline \multicolumn{13}{|c|}{ Time from grip force to load force onsets (msec) } \\
\hline Comparison subjects & 62 & 8 & 75 & 12 & 81 & 10 & 74 & 11 & 74 & 10 & 70 & 10 \\
\hline Patients & 114 & 7 & 147 & 11 & 148 & 9 & 131 & 9 & 135 & 8 & 142 & 9 \\
\hline \multicolumn{13}{|c|}{ Time from load force to grip force peaks (msec) } \\
\hline Comparison subjects & 89 & 16 & 96 & 16 & 115 & 15 & 62 & 13 & 102 & 15 & 135 & 19 \\
\hline Patients & 85 & 14 & 128 & 14 & 120 & 13 & 62 & 11 & 119 & 13 & 152 & 17 \\
\hline \multicolumn{13}{|c|}{ Peak grip force (newtons [N]) } \\
\hline Comparison subjects & 10.1 & 0.5 & 15.2 & 0.6 & 18.7 & 0.8 & 10.9 & 0.5 & 15.1 & 0.6 & 17.9 & 0.7 \\
\hline Patients & 11.6 & 0.4 & 16.5 & 0.5 & 22.2 & 0.8 & 12.7 & 0.5 & 17.2 & 0.5 & 20.4 & 0.6 \\
\hline \multicolumn{13}{|c|}{ Peak rate of change of grip force ( $\mathrm{N} / \mathrm{sec})$} \\
\hline Comparison subjects & 78 & 4 & 116 & 6 & 132 & 8 & 87 & 5 & 114 & 6 & 125 & 7 \\
\hline Patients & 78 & 4 & 104 & 5 & 132 & 7 & 87 & 4 & 111 & 5 & 116 & 6 \\
\hline
\end{tabular}

${ }^{a}$ With their dominant hand, subjects used a precision grip between the thumb and four fingers to grasp a manipulandum with wood-surfaced sides $(4.5 \times 10 \times 9 \mathrm{~cm}, 450 \mathrm{~g})$. A six-axis load cell was mounted within the manipulandum to measure forces and torques normal and tangential to the loading axes. Data were sampled at $1000 \mathrm{~Hz}$. In the lift task, subjects reached for and lifted the manipulandum, maintained it at a steady position $20 \mathrm{~cm}$ from the tabletop for approximately 3 seconds, and placed it back on the table. The manipulandum was covered with increasingly slippery material (sandpaper, cotton, silk) to vary the texture of the grasp surface, and iron bars were attached to the manipulandum to vary the mass. Subjects performed five trials for each texture/mass combination, presented in randomized blocks.

${ }^{\mathrm{b}}$ Light mass $=450 \mathrm{~g}$; medium mass $=710 \mathrm{~g}$; heavy mass $=880 \mathrm{~g}$.

FIGURE 3. Grip Force Used by 16 Healthy Comparison Subjects and 16 Patients With Schizophrenia to Hold Objects of Various Textures and Masses

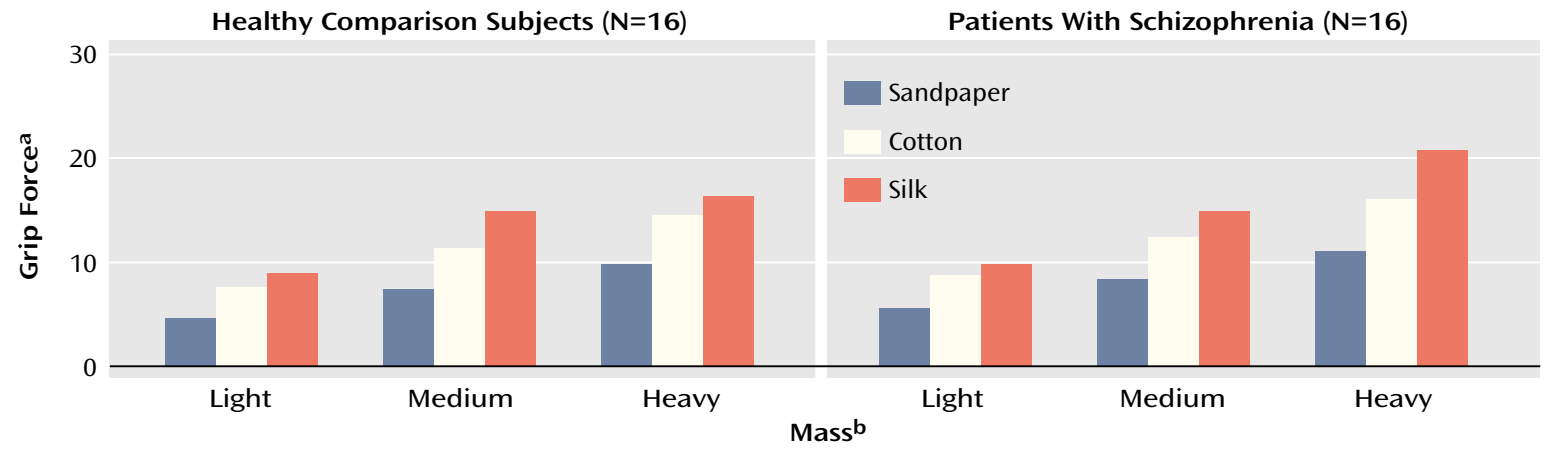

${ }^{a}$ Forces measured in newtons $(\mathrm{N})$.

${ }^{\mathrm{b}}$ Light mass $=450 \mathrm{~g}$; medium mass $=710 \mathrm{~g}$; heavy mass $=880 \mathrm{~g}$.

comparison subjects. The statistical analysis showed that the group effect was significant neither for pendulum accuracy $(\mathrm{F}=2.89, \mathrm{df}=1,547, \mathrm{n} . \mathrm{s}$.) nor for the produced force of impact, i.e., load force peak ( $\mathrm{F}=0.02$, $\mathrm{df}=1,547$, n.s.).

For the patient group, the statistical analyses revealed a shift in the timing of the anticipatory grip force responses. The patients started increasing their grip force levels too late in respect to the start of the arm movement. As a consequence 1) the time from the onset of the grip force increase to impact (i.e., peak load force) was significantly shorter for the patients than for the comparison subjects $(\mathrm{F}=12.90, \mathrm{df}=1,485, \mathrm{p}<0.01)$, and 2) the grip force peak occurred significantly later after impact for the patients than for the comparsion subjects $(\mathrm{F}=20.47, \mathrm{df}=1,547, \mathrm{p}<0.01)$.

On average, grip force baselines were significantly higher $(\mathrm{F}=21.44, \mathrm{df}=1,547, \mathrm{p}<0.01)$ and more variable $(\mathrm{F}=$ 11.16, $\mathrm{df}=1,547, \mathrm{p}<0.01$ ) for the patients than for the comparison subjects. To standardize across groups, grip force baseline was thus subtracted from the overall grip force levels in the following analyses. Grip force peak was scaled to load force peak $(\mathrm{F}=196.23, \mathrm{df}=1,547, \mathrm{p}<0.01)$, and this adjustment was similar for the patients and the comparison subjects $(\mathrm{F}=2.91, \mathrm{df}=1,547$, n.s.). However, the interaction of group and target was significant $(\mathrm{F}=7.24, \mathrm{df}=1,547$, $\mathrm{p}<0.01$ ), indicating that the comparison subjects had larger grip force peaks than the patients when aiming for the $18^{\circ}$ target. This is very probably a ceiling effect, as the patients had significantly higher grip force baseline levels. All subjects increased grip force at a higher rate when planning to produce larger load force peaks $(\mathrm{F}=202.50, \mathrm{df}=$ $1,547, \mathrm{p}<0.01)$. Grip force was increased at a faster rate for the comparison subjects than for the patients $(\mathrm{F}=4.28, \mathrm{df}=$ $1,547, \mathrm{p}<0.05)$.

\section{Resist Task}

All subjects maintained a steady grip force baseline while waiting for the auditory tone. As they saw the pendulum fall, the subjects increased their grip force levels, 
TABLE 2. Measures of Grip Force and Load Force for 16 Healthy Comparison Subjects and 16 Patients With Schizophrenia Who Completed Collision Tasks Involving Producing or Resisting a Collision With a Pendulum ${ }^{\mathrm{a}}$

\begin{tabular}{|c|c|c|c|c|c|c|c|c|c|c|c|c|}
\hline \multirow[b]{3}{*}{ Measure and Group } & \multicolumn{6}{|c|}{ Pendulum Target Angle of Hit Task } & \multicolumn{6}{|c|}{ Pendulum Release Angle of Resist Task } \\
\hline & \multicolumn{2}{|c|}{$5^{\circ}$} & \multicolumn{2}{|c|}{$10^{\circ}$} & \multicolumn{2}{|c|}{$18^{\circ}$} & \multicolumn{2}{|c|}{$6^{\circ}$} & \multicolumn{2}{|c|}{$12^{\circ}$} & \multicolumn{2}{|c|}{$20^{\circ}$} \\
\hline & Mean & SD & Mean & SD & Mean & SD & Mean & SD & Mean & SD & Mean & SD \\
\hline \multicolumn{13}{|c|}{ Peak load force (newtons [N]) } \\
\hline Comparison subjects & 3.2 & 0.5 & 8.9 & 0.5 & 25.4 & 0.6 & 3.2 & 0.8 & 8.4 & 2.3 & 26.5 & 5.9 \\
\hline Patients & 3.2 & 0.4 & 8.7 & 0.5 & 25.6 & 0.5 & 3.5 & 0.9 & 8.3 & 2.8 & 26.5 & 8.6 \\
\hline \multicolumn{13}{|c|}{$\begin{array}{l}\text { Time from grip force onset to load force } \\
\text { peak (msec) }\end{array}$} \\
\hline Comparison subjects & 217 & 17 & 250 & 17 & 322 & 19 & 141 & 31 & 202 & 30 & 234 & 28 \\
\hline Patients & 169 & 16 & 215 & 17 & 256 & 16 & 191 & 34 & 215 & 25 & 199 & 25 \\
\hline \multicolumn{13}{|c|}{$\begin{array}{l}\text { Time from load force to grip force peaks } \\
\text { (msec) }\end{array}$} \\
\hline Comparison subjects & 36 & 5 & 27 & 5 & 16 & 5 & 84 & 7 & 89 & 7 & 92 & 7 \\
\hline Patients & 57 & 4 & 48 & 5 & 27 & 5 & 91 & 9 & 93 & 6 & 99 & 6 \\
\hline \multicolumn{13}{|l|}{ Baseline grip force $(\mathrm{N})$} \\
\hline Comparison subjects & 7.8 & 0.1 & 8.1 & 0.2 & 8.6 & 0.1 & 11.1 & 0.1 & 11.7 & 0.1 & 12.5 & 0.2 \\
\hline Patients & 9.7 & 0.2 & 9.3 & 0.2 & 10.9 & 0.5 & 12.3 & 0.2 & 10.8 & 0.3 & 10.9 & 0.2 \\
\hline \multicolumn{13}{|l|}{ Peak grip force $(\mathrm{N})$} \\
\hline Comparison subjects & 6.1 & 0.9 & 15.2 & 1.0 & 28.7 & 1.1 & 6.2 & 1.4 & 12.8 & 1.4 & 21.8 & 1.3 \\
\hline Patients & 7.1 & 0.8 & 15.9 & 0.9 & 23.0 & 1.0 & 7.3 & 1.5 & 13.4 & 1.2 & 22.8 & 1.2 \\
\hline \multicolumn{13}{|c|}{ Peak rate of change of grip force $(\mathrm{N} / \mathrm{sec})$} \\
\hline Comparison subjects & 53 & 7 & 124 & 8 & 218 & 9 & 48 & 16 & 99 & 15 & 182 & 14 \\
\hline Patients & 49 & 7 & 117 & 7 & 191 & 7 & 55 & 17 & 126 & 13 & 167 & 13 \\
\hline
\end{tabular}

a Subjects stood facing a pendulum (an aluminum bar, $75 \mathrm{~cm}$ in length and $750 \mathrm{~g}$ in mass) that swung in the frontoparallel plane. A circular potentiometer located at the pivoting point measured the angle of pendulum swing (in degrees). Subjects gripped the light (450 g), cottoncovered manipulandum between the thumb and four fingers of their dominant hand. In the hit task, the subject used the manipulandum to hit the head of the pendulum to make it reach a target height of $5^{\circ}, 10^{\circ}$, or $18^{\circ}\left( \pm 0.5^{\circ}\right)$. In the resist task, the subject held the manipulandum against the head of the pendulum and attempted to maintain it immobile when the pendulum was raised to a preset angle of $6^{\circ}, 12^{\circ}$, or $20^{\circ}\left( \pm 0.5^{\circ}\right)$. Each subject performed 12 trials for each pendulum target angle and for each pendulum release angle.

which reached a maximum value soon after the time of impact (Table 2; Figure 2, right).

The time from the onset of grip force increase to load force peak was similar for the patients and the comparison subjects $(\mathrm{F}=0.16, \mathrm{df}=2,565$, n.s.). The time between load force and grip force peaks was also similar for the patients and the comparison subjects $(\mathrm{F}=2.67, \mathrm{df}=2,565$, n.s.). For all subjects, grip force levels were increased at a faster rate $(\mathrm{F}=70.42, \mathrm{df}=2,565, \mathrm{p}<0.01)$ and reached higher peaks $(\mathrm{F}=$ 33.33, $\mathrm{df}=2,565, \mathrm{p}<0.01$ ) for bigger load force peaks. Grip force was increased at a similar rate $(\mathrm{F}=0.25, \mathrm{df}=2,565$, n.s.) and reached similar peak levels $(\mathrm{F}=1.74, \mathrm{df}=2,565$, n.s.) for the patients and the comparison subjects.

\section{Discussion}

A systematic procedure was used to establish the nature of the motor deficits observed in patients with schizophrenia. Specifically, three experimental tasks were selected to assess whether schizophrenia affects the predictive mechanisms required for the scaling, timing, and/or sequencing of motor actions.

During object lift, the patients and the comparison subjects increased grip force levels at a similar rate. However, the patients had a significant delay between the onset of grip closure and the start of the upward lift movement. This dyscoordination force pattern may explain why the patients developed excessive force levels compared to the comparison subjects. In the hit task, patients started to increase grip force levels after the start of the arm move- ment; thus, grip force responses peaked systematically after impact. This was in marked contrast to the pattern observed in the healthy subjects. The two control deficits described here may have been related to a problem in the timing of the occurrence of an event, i.e., object liftoff or time of impact. It could also have been a problem in the sequencing of multiple subgoals within a movement. Consequently, to partial out these two confounds, subjects were presented with a third task in which they were asked to increase grip force levels in time for an externally imposed collision. In this task, the timing of the grip force peaks was similar for the patients and the comparison subjects, indicating that patients with schizophrenia were not impaired in a motor task for which only the timing of an external event was necessary. Taken together, these findings show that patients with schizophrenia were specifically impaired in the fluid sequencing of motor actions.

In contrast, the predictive scaling of motor responses was not impaired in patients with schizophrenia. In the lift task, grip force levels were scaled both to the weight and to the texture of the object. The rates of grip force development were also adjusted, revealing that grip force was set to the final force level right from the start of grip closure. These results indicate 1) that the integrative mechanisms involved in the identification of object attributes for object-oriented movement are preserved in patients with schizophrenia, and 2) that patients with schizophrenia are not impaired in the predictive scaling of force levels. This later point was further emphasized in the collision tasks for which sensorimotor feedback mechanisms were of no 
use. In both the hit and the resist tasks, grip force rates and peaks were scaled to the force of impact in a similar fashion for the patients and the comparison subjects. These results do not support the supposed deficit in the central monitoring of action in schizophrenia $(7,8,12)$. Our findings are, however, in accordance with the findings of experiments examining the automatic control of upper limb movements, in which patients with schizophrenia were shown to scale movement parameters in reaching and pointing tasks $(13,14)$ and to adjust grip force levels in a predictive manner for externally and self-imposed collisions (15).

The data reported here demonstrate that schizophrenia is not associated with an overall problem in the predictive control of movement but rather with a specific deficit in the fluid sequencing of motor actions. Functional brain imaging studies have pointed to the cerebellum and the basal ganglia as major brain areas involved in the automatic control of sequential movements (16). Both of these brain regions are thought to be dysfunctional in patients with schizophrenia $(17,18)$.

The following discussion considers three alternative explanations for the origins of the sequencing deficits observed in the patients in this study.

First, striatal dysregulation in patients with schizophrenia has been frequently associated with neuroleptic treatments, which sometimes induce Parkinson-like symptoms such as resting tremor, reduced movement speed, and posture/balance impairments. However, in the patients in this study, none of these symptoms were observed. Furthermore, identical motor performance in the three experimental tasks was exhibited by both the patients who had received neuroleptic medication and by those who had not. Previous clinical observations detected dysfunctional motor sequencing (dysdiadochokinesia) as early as 1919 (2), i.e., well before the introduction of chlorpromazine. For these various reasons, we feel that our results are not related to long-term use of neuroleptic drugs. To further confirm the absence of treatment effects on movement sequencing, future research should focus on testing patients who have never received neuroleptic medication.

A second hypothesis is that the sequencing deficits observed in the patients with schizophrenia are due to isolated dysfunctions of either the basal ganglia or the cerebellum, or of both structures. Neuropsychological studies using the lift task have shown that both patients with Parkinson's disease (19) and those with cerebellar lesions (20) display a prolonged latency between the onsets of grip and load force increases. These dyscoordination force patterns are similar to those observed in the patients with schizophrenia in this study. However, both cerebellar lesion patients and Parkinson's disease patients are also characterized by a marked slowness in the rate of grip closure, a feature that was not observed in the patients in this study. Overall, these observations suggest that schizo- phrenia is not associated with isolated dysfunction to these two specific brain areas.

Third, it is possible that the pathology is due to an abnormality in the neural circuitry involving distributed and specific neural substrates. The cortico-cerebellar-thalamic-cortical circuit has been suggested as a possible substrate for the fluid sequencing of movement (21), as it enables very rapid on-line feedback between the cerebral cortex and the cerebellum. It is interesting to note that results obtained in a series of neuroimaging studies on memory deficits in patients with schizophrenia led Andreasen (22) to propose the cortico-cerebellar-thalamiccortical circuit as the possible neural substrate for the cognitive dysmetria theory. This neo-Bleulerian model presents schizophrenia as a fundamental cognitive abnormality that reflects a disorder in the specific neural circuits underlying the control of "synchrony," a mechanism necessary for the fluid coordination of sequences of motor activities and thought. The motor behavior data reported in the present study fit well within the dysmetria theoretical framework. However, further behavioral and functional imaging studies are required to confirm the central role of the cortico-cerebellar-thalamic-cortical circuit in the pathology of schizophrenia. Furthermore, for the validation of the cognitive dysmetria theory, a systematic correlation between the severity of motor and cognitive deficits would be essential.

Received Jan. 29, 2002; revision received July 11, 2002; accepted July 18, 2002. From Institut National de la Santé et de la Recherche Médicale. Address reprint requests to Dr. Delevoye-Turrell, Institut National de la Santé et de la Recherche Médicale, U405-HUS, 1 PI. de l'Hôpital, 67091 Strasbourg, France; yvonne.delevoye@laposte.net (e-mail).

Supported by the program Alliance and by the Institut National de la Santé et de la Recherche Médicale (Dr. Delevoye-Turrell). The authors thank F. Kaufman and Drs.P. Sonntag, M. Lacambre, M.A. Zimmerman, P. Vidailhet, M. Kazes, E. Reber, and M. Riutort for referring the patients and P. Gries and S. Allen for technical support.

\section{References}

1. Bleuler E: Dementia Praecox or the Group of Schizophrenias (1908). Translated by Zinkin J. New York, International Universities Press, 1950

2. Kraepelin E: Dementia Praecox and Paraphrenia (1919). Translated by Barclay RM; edited by Robertson GM. New York, Robert E Krieger, 1971

3. Boks MPM, Russo S, Knegtering R, van den Bosch RJ: The specificity of neurological signs in schizophrenia: a review. Schizophr Res 2000; 43:109-116

4. Sullivan EV, Shear PK, Zipursky RB, Sagar HJ, Pfefferbaum A: A deficit profile of executive, memory, and motor functions in schizophrenia. Biol Psychiatry 1994; 36:641-653

5. Johansson RS, Cole KJ: Grasp stability during manipulative actions. Can J Physiol Pharmacol 1994; 72:511-524

6. Flanagan JR, Wing AM: The role of internal models in motion planning and control: evidence from grip force adjustments during movements of hand-held loads. J Neurosci 1997; 17: 1519-1528

7. Feinberg I, Guazzelli M: Schizophrenia-a disorder of the corollary discharge systems that integrate the motor systems of 
thought with the sensory systems of consciousness. Br J Psychiatry 1999; 174:196-204

8. Frith C, Rees G, Friston K: Psychosis and the experience of self: brain systems underlying self-monitoring. Ann NY Acad Sci 1998; 15:170-178

9. Johansson RS, Westling G: Roles of glabrous skin receptors and sensorimotor memory in automatic control of precision grip when lifting rougher or more slippery objects. Exp Brain Res 1984; 56:550-564

10. Turrell YN, Li F-X, Wing AM: Grip force dynamics in the approach to a collision. Exp Brain Res 1999; 128:86-91

11. Turrell YN, Li FX, Wing AM: Evaluating the minimum grip force required when grasping objects under impulsive loading conditions. Behav Res Methods Instrum Comput 2001; 33:38-45

12. Frith C, Blakemore SJ, Wolpert DM: Abnormalities in the awareness and control of action. Phil Trans R Soc Lond B 2000; 355: 1771-1788

13. Carnahan H, Elliott D, Velamoor VR: Influence of object size on prehension in leukotomized and unleukotomized individuals with schizophrenia. J Clin Exp Neuropsychol 1996; 8:136-147

14. Saoud M, Coello Y, Dumas P, Franck N, D'Amato T, Dalery J, Rossetti Y: Visual pointing and speed/accuracy trade-off in schizophrenia. Cognitive Neuropsychiatry 2000; 5:123-134
15. Delevoye-Turrell Y, Giersch A, Danion JM: A deficit in the adjustment of grip force responses in schizophrenia. Neuroreport 2002; 13:1537-1539

16. Tanji J: Sequential organization of multiple movements: involvement of cortical motor areas. Annu Rev Neurosci 2001; 24:631-651

17. Menon V, Anagnoson RT, Glover GH, Pfefferbaum A: Functional magnetic resonance imaging evidence for disrupted basal ganglia function in schizophrenia. Am J Psychiatry 2001; 158:646649

18. Wiser AK, Andreasen NC, O'Leary DS, Watkins GL, Boles Ponto LL, Hichwa RD: Dysfunctional cortico-cerebellar circuits cause "cognitive dysmetria" in schizophrenia. Neuroreport 1998; 9: 1895-1899

19. Fellows SJ, Noth J, Schwarz M: Precision grip and Parkinson's disease. Brain 1998; 121:1771-1784

20. Müller F, Dichgans J: Dyscoordination of pinch and lift forces during grasp in patients with cerebellar lesions. Exp Brain Res 1994; 101:485-492

21. Kandel ER, Schwartz JH, Jessell TM: Principles of Neural Science, 3rd ed. New York, Elsevier, 1991

22. Andreasen NC: A unitary model of schizophrenia: Bleuler's "fragmented phrene" as schizencephaly. Arch Gen Psychiatry 1999; 56:781-787 